\title{
CONSTITUINTES QUÍMICOS VOLÁTEIS E NÃO-VOLÁTEIS DE Cochlospermum vitifolium (WILLDENOW) SPRENGEL
}

\author{
Sheyla Cristiane Xenofonte de Almeida, Telma Lêda Gomes de Lemos, Edilberto Rocha Silveira e Otília Deusdênia Loiola \\ Pessoa* \\ Departamento de Química Orgânica e Inorgânica, Centro de Ciências, Universidade Federal do Ceará, CP 12200, 60021-970 \\ Fortaleza - CE
}

Recebido em 3/2/04; aceito em 5/7/04; publicado na web em 5/11/04

\begin{abstract}
VOLATILE AND NON-VOLATILE CHEMICAL CONSTITUENTS OF Cochlospermum vitifolium (WILLDENOW) SPRENGEL. The essential oils from leaves, root bark and root wood of Cochlospermum vitifolium were investigated for the first time. The oils were obtained by hydrodistillation and analyzed by GC/MS. The main volatile constituents were $\beta$-caryophyllene (8.2 46.5\%), $\beta$-bisabolene (11.5 - 29.3\%), $\gamma$-muurolene (28.4\%), $\alpha$-humulene (26.0\%), 1-hydroxy-3-hexadecanone $(16.2-19.5 \%)$ and $\beta$-pinene (10.6\%). Phytochemical analysis of the root bark and root wood extracts yielded excelsin, pinoresinol, narigenin, aromadendrin, galic acid and a triacylbenzene, along with $\beta$-sitosterol and stigmasterol and their D-glucosides. The structures of all compounds were determined by analyses of the spectroscopic data (NMR and MS), and comparison with the literature.
\end{abstract}

Keywords: Cochlospermum vitifolium; Cochlospermaceae; chemical composition.

\section{INTRODUÇÃO}

A família Cochlospermaceae compreende apenas dois gêneros, Cochlospermum e Amoreuxia, os quais reúnem cerca de quinze espécies distribuídas principalmente nas regiões tropicais do globo terrestre, especialmente nas Américas e África. A principal diferença entre os dois taxons encontra-se na simetria da flor, a qual é actinomórfica em Cochlospermum e zigomórfica em Amoreuxia ${ }^{1}$.

O gênero Cochlospermum, de distribuição pantropical, é constituído por aproximadamente onze espécies, quatro das quais, $C$. angolense, C. planchonii, C. tinctorium e C. vitifolium, apresentam significante importância medicinal ${ }^{1-6}$. A investigação química de plantas do gênero tem resultado no isolamento de metabólitos secundários pertencentes a várias classes estruturais como flavonóides, apocarotenóides, acetogeninas, triacilbenzenos, triterpenos e esteróides $^{4,7-11}$.

A ocorrência freqüente de $C$. vitifolium, aliada à ausência de estudos químicos, exceto por um trabalho que relata o isolamento de dois apocarotenóides ${ }^{12}$, motivou-nos a investigar a constituição química da referida espécie. C. vitifolium é uma pequena árvore, popularmente conhecida como algodão-bravo ou algodão do campo em diversos estados do país, e como pacotê, no Ceará. Floresce no período em que as folhas caem, de dezembro a março, no hemisfério Norte, e de junho a outubro, abaixo da linha do equador ${ }^{1}$. No estado do Ceará, a época de floração coincide com o período de total estiagem, quando a paisagem se apresenta completamente árida. Neste cenário, $C$. vitifolium encontra-se totalmente despida de folhagem, entretanto, suas flores grandes e vistosas, em cachos terminais de coloração amarelo-dourado, enfeitam os sertões cearenses. Em diversos países, como Cuba, Costa Rica, Guatemala e México, a referida espécie é de importância etnofarmacológica, sendo utilizada principalmente no tratamento de malária, icterícia, úlcera e doenças renais, hepáticas e respiratórias, além de possuir propriedades emenagogas 5 .

*e-mail: opessoa@ufc.br
Este trabalho descreve os resultados obtidos na investigação química de $C$. vitifolium, envolvendo a identificação dos componentes químicos voláteis, os quais estão sendo divulgados pela primeira vez, bem como o isolamento e a caracterização dos constituintes químicos não-voláteis.

\section{RESULTADOS E DISCUSSÃO}

Iniciou-se a investigação química de $C$. vitifolium com o estudo dos óleos essenciais obtidos a partir de folhas, casca e lenho da raiz. Os componentes químicos identificados, incluindo as percentagens e os índices de Kovat, encontram-se descritos na Tabela 1. No óleo essencial das folhas foram identificados dez componentes, entre monoterpenos e sesquiterpenos, representando $99,9 \%$ da constituição volátil. Os principais constituintes detectados foram $\beta$-cariofileno (46,5\%), $\alpha$-humuleno (26,0\%) e $\beta$-pineno (10,6\%). Ao contrário do óleo essencial das folhas, nos óleos extraídos da casca e do lenho da raiz nenhum monoterpeno foi detectado, por outro lado foram identificadas cetonas alifáticas de cadeia longa. Nos óleos obtidos da casca e lenho da raiz foram identificados um total de 88,0 e 90,0\% dos constituintes químicos, respectivamente. Exceto pela presença de $\gamma$ muuroleno, o qual constitui $28,4 \%$ do óleo essencial do lenho da raiz, os componentes majoritários presentes em ambos os óleos foram $\beta$ bisaboleno (11,5 - 29,3\%), 1-hidroxi-3-hexadecanona (16,2 - 19,5\%) e $\beta$-cariofileno $(8,2-11,6 \%)$. Cetonas alifáticas, inclusive hidroxiladas, foram previamente identificadas nos óleos essenciais de $C$. tinctorium e C. planchonii ${ }^{9,13}$ estando, portanto, nossos resultados em acordo com a composição química volátil registrada para plantas do gênero.

A investigação das frações $\mathrm{CHCl}_{3}$ e AcOEt, provenientes do fracionamento cromatográfico do extrato $\mathrm{EtOH}$ da casca da raiz resultou em isolamento e caracterização das lignanas excelsina $(\mathbf{1})^{14} \mathrm{e}$ pinoresinol (2) ${ }^{15}$; da mistura binária dos esteróides $\beta$-sitosterol (3) e estigmasterol (4) na proporção de 3:1; das flavanonas naringenina (5) e aromadendrina (6) ${ }^{16}$; da mistura constituída de 3-O- $\beta$ glicopiranosil- $\beta$-sitosterol (7) e 3-O- $\beta$-glicopiranosil-estigmasterol $(8)^{17}$, além do ácido gálico $(9)^{18}$. O extrato EtOH do lenho da raiz foi submetido a procedimentos cromatográficos semelhantes àqueles 
Tabela 1. Constituintes químicos voláteis identificados nos óleos essenciais de folhas (I), casca (II) e lenho (III) da raiz de C. vitifolium

\begin{tabular}{|c|c|c|c|c|}
\hline Constituintes & IK & $I^{*}$ & II $^{*}$ & $1 \mathrm{II}^{*}$ \\
\hline$\alpha$-pineno & 936 & 4,8 & - & - \\
\hline$\beta$-pineno & 975 & 10,6 & - & - \\
\hline mirceno & 991 & 1,7 & - & - \\
\hline$\beta$-felandreno & 1025 & 1,7 & - & - \\
\hline$E$ - $\beta$-ocimeno & 1048 & 2,7 & - & - \\
\hline$\alpha$-terpineno & 1197 & 1,6 & - & - \\
\hline$\alpha$-copaeno & 1368 & - & 0,9 & - \\
\hline$\beta$-cariofileno & 1418 & 46,5 & 8,2 & 11,6 \\
\hline$\alpha$-humuleno & 1450 & 26,0 & 1,3 & 1,9 \\
\hline$E$ - $\beta$-farneseno & 1458 & - & 3,7 & 2,7 \\
\hline$\gamma$-muuroleno & 1480 & - & - & 28,4 \\
\hline D-germacreno & 1485 & 3,3 & 3,4 & - \\
\hline 2-dodecanona & 1496 & - & 1,2 & 6,3 \\
\hline 2-tridecanona & 1499 & - & 3,2 & 2,4 \\
\hline$\beta$-bisaboleno & 1512 & - & 29,3 & 11,5 \\
\hline$Z$ - $\gamma$-bisaboleno & 1523 & - & 1,2 & - \\
\hline$\delta$-cadineno & 1525 & - & 2,2 & 2,8 \\
\hline$E$ - $\gamma$-bisaboleno & 1531 & - & 2,1 & 0,8 \\
\hline óxido de cariofileno & 1575 & 1,0 & - & - \\
\hline 3-tetradecanona & 1589 & - & 0,9 & - \\
\hline epi- $\alpha$-cadinol & 1638 & - & 0,9 & 0,9 \\
\hline epi- $\alpha$-muurolol & 1641 & - & 1,4 & 1,6 \\
\hline$\alpha$-muurolol & 1644 & - & - & 0,6 \\
\hline$\alpha$-cadinol & 1653 & - & 2,3 & 2,6 \\
\hline epi- $\alpha$-bisabolol & 1680 & - & 3,2 & - \\
\hline 1-hidroxi-3-tetradecanona & 1785 & - & 3,1 & - \\
\hline 1-hidroxi-3-hexadecanona & 1922 & - & 19,5 & 16,2 \\
\hline Total & & 99,9 & 88,0 & 90,3 \\
\hline
\end{tabular}

*Teor baseado na porcentagem normalizada.

utilizados para a casca da raiz. Todas as frações em estágio de semipurificação foram monitoradas por CCD, e todos os compostos previamente isolados, detectados. Uma alíquota da fração $\mathrm{CHCl}_{3}$ do lenho foi submetida à hidrólise alcalina seguida de metilação e posteriormente analisada por cromatografia gás-líquido acoplada à espectrometria de massa, possibilitando a identificação dos ácidos láurico $\left(\mathrm{C}_{12}: 0 ; 5,2 \%\right)$, mirístico $\left(\mathrm{C}_{14}: 0 ; 5,5 \%\right)$, palmítico $\left(\mathrm{C}_{16}: 0\right.$; $36,5 \%)$, linoléico $\left(\mathrm{C}_{18}: 12 ; 14,5 \%\right)$, oléico $\left(\mathrm{C}_{18}: 1 ; 23,8 \%\right)$ e esteárico $\left(\mathrm{C}_{18}: 0 ; 6,0 \%\right)$. O triacilbenzeno, caracterizado como 1-dodecanoil3,5-di(tetradecanoil)benzeno $(\mathbf{1 0})^{19}$, foi isolado, após sucessivas cromatografias, da fração insaponificável.

As estruturas de todos os compostos isolados foram caracterizadas através de métodos espectroscópicos como IV, EM e RMN ${ }^{1} \mathrm{H}$ e ${ }^{13} \mathrm{C}$, uni- e bidimensional, e confirmadas através da comparação de seus dados físicos e espectrais com aqueles registrados na literatura. A análise dos componentes voláteis, assim como dos ésteres metílicos correspondentes aos ácidos graxos descritos acima, foi realizada por CG/EM. A identificação dos constituintes foi realizada comparando-se o espectro de massa de cada componente individual com espectros de massa armazenados em um banco de dados ${ }^{20,21}$, bem como por comparação com espectros de massa disponíveis na literatura ${ }^{22,23}$, levando-se em conta principalmente o padrão de fragmentação e o índice de Kovat.

\section{CONCLUSÃO}

Tendo como base informações colhidas através de um levantamento bibliográfico exaustivo foi possível estabelecer um perfil sobre<smiles>COc1cc(C2OCC3C(c4cc(OC)c5c(c4)OCO5)OCC23)cc2c1OCO2</smiles><smiles>COc1cc(O)cc(C2OCC3C(c4ccc(O)c(OC)c4)OCC23)c1</smiles><smiles>CCC(CCC(C)C1CCC2C1CCC1C3(C)CCC(O)C(C)(C)C3=CCC21C)C(C)C</smiles><smiles>O=C1CC(c2ccc(O)cc2)Oc2cc(O)cc(O)c21</smiles>

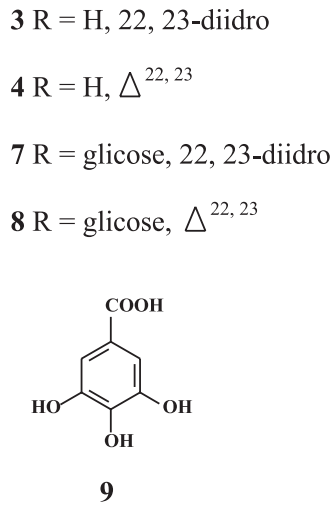<smiles>O=C1c2c(O)cc(O)cc2OC(c2ccc(O)cc2)C1O</smiles>

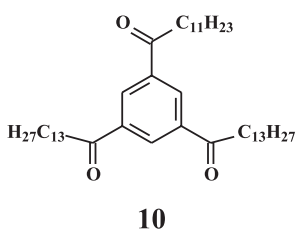

a constituição química volátil e não-volátil das plantas que compõem o táxon Cochlospermum. Os óleos essenciais de algumas espécies do referido gênero têm sido analisados, mostrando-se ricos em sesquiterpenos e cetonas alifáticas de cadeia longa. Triacilbenzenos e flavonóides aparecem dentre os constituintes químicos mais freqüentemente isolados. Assim, a composição química de C. vitifolium, assemelha-se à de outras espécies do gênero, exceto pela obtenção de lignanas. $\mathrm{O}$ isolamento de lignóides relatados neste trabalho pela primeira vez, a partir de uma espécie do gênero, é de relevante importância, em virtude das muitas atividades que lhes são atribuídas.

\section{PARTE EXPERIMENTAL}

\section{Procedimentos experimentais gerais}

Os pontos de fusão foram determinados em equipamento da Micro Química, modelo MQAFP 301. Os espectros de absorção no IV foram obtidos em espectrômetro Perkin-Elmer, modelo 1000-FT, utilizando-se pastilhas de $\mathrm{KBr}$. Os espectros de $\mathrm{RMN}{ }^{1} \mathrm{H}$ e ${ }^{13} \mathrm{C}$, unie bidimensionais, foram obtidos em espectrômetros Bruker, Modelos DPX-300 (300 MHz para ${ }^{1} \mathrm{H}$ e $75 \mathrm{MHz}$ para ${ }^{13} \mathrm{C}$ ) e Avance DRX$500\left(500 \mathrm{MHz}\right.$ para ${ }^{1} \mathrm{H}$ e $125 \mathrm{MHz}$ para $\left.{ }^{13} \mathrm{C}\right)$. Os espectros de massa das substâncias isoladas foram obtidos por impacto eletrônico a 70 eV, em espectrômetro de baixa resolução Shimadzu, modelo QP 5000, DI-50, enquanto os espectros de massa dos constituintes voláteis foram obtidos em espectrômetro Hewlett-Packard, modelo HP-5971 A, acoplado a cromatógrafo gás-líquido, modelo HP-5890 A, série II (CG/EM), provido de coluna capilar apolar DB-5 (30 m de comprimento $\mathrm{x} 0,25 \mathrm{~mm}$ de diâmetro interno), utilizando hélio como gás de arraste e um gradiente crescente de temperatura de $4{ }^{\circ} \mathrm{C} / \mathrm{min}$ de 50 a $180{ }^{\circ} \mathrm{C}$, e $20{ }^{\circ} \mathrm{C} / \mathrm{min}$ de 180 a $280{ }^{\circ} \mathrm{C}$, sendo a temperatura do injetor de $250{ }^{\circ} \mathrm{C}$ e a do detetor de $200{ }^{\circ} \mathrm{C}$. 
Nas cromatografias de adsorção em coluna (CC) foi utilizado gel de sílica 60 (230 - 400 mesh), enquanto para as cromatografias de camada delgada analítica (CCDA) e preparativa (CCDP) foram utilizadas cromatoplacas de gel de sílica, 1.05735, $60 \mathrm{~A}^{\circ}$, com indicador de fluorescência na faixa de $254 \eta \mathrm{m}$ (Merck) e gel de sílica $\mathrm{S}$ da Carlo Erba (cod. 45335), respectivamente. As substâncias foram reveladas sob luz ultravioleta (254 e 366 ๆm) e/ou pela aspersão com solução de vanilina/ácido perclórico/EtOH, seguida de aquecimento, ou ainda pela exposição a vapores de iodo.

\section{Material vegetal}

C. vitifolium foi coletada em março de 2002, na localidade de Antônio Diôgo, município de Baturité - CE. A exsicata correspondente à coleta da planta encontra-se depositada no Herbário Prisco Bezerra (EAC), sob o registro 31.419. A identificação botânica foi realizada pelo Prof. E. P. Nunes do Departamento de Biologia - UFC.

\section{Extração e isolamento}

As raizes de $C$. vitifolium foram separadas em casca (600 g) e lenho (1680 g), moídas, secas à temperatura ambiente, e cada uma das partes submetida à percolação em EtOH, resultando nos respectivos extratos, após evaporação dos solventes sob pressão reduzida. Os extratos brutos, casca (54 g) e lenho $(65 \mathrm{~g})$, foram fracionados em gel de sílica obtendo-se as seguintes frações: casca $\left[\mathrm{CHCl}_{3}(2,0 \mathrm{~g})\right.$, AcOEt $(9,1 \mathrm{~g})$, acetona $(10,9 \mathrm{~g})$ e $\mathrm{MeOH}(25,1)$; lenho $\left[\mathrm{CHCl}_{3}(6,2\right.$ g), $\operatorname{AcOEt}(15,4 \mathrm{~g})$, acetona $(8,2 \mathrm{~g})$ e $\mathrm{MeOH}(23,5)]$. A fração $\mathrm{CHCl}_{3}$ $(2,0 \mathrm{~g})$ foi cromatografada sobre $31,0 \mathrm{~g}$ de gel de sílica e eluída com hexano, hexano/AcOEt em misturas de polaridade crescente, AcOEt e MeOH. As frações colhidas foram analisadas em CCD e posteriormente reunidas em 10 subfrações. A subfração hexano/AcOEt (7:3) apresentou um precipitado amarelo claro, o qual foi filtrado e recristalizado em AcOEt, resultando no isolamento de $\mathbf{1}$ (26 mg). A subfração hexano/AcOEt (6:4) foi recromatografada em gel de sílica, utilizando os mesmos eluentes, e posteriormente submetida à CCDP, usando $\mathrm{CHCl}_{3}$ como eluente, obtendo-se o composto 2 (13 mg). Na subfração hexano/AcOEt (1:1) houve a formação de cristais em forma de agulhas, os quais foram filtrados e recristalizados em $\mathrm{MeOH}$, resultando no isolamento de uma mistura binária constituída de $\mathbf{3}$ e $\mathbf{4}$ (12 mg). A fração AcOEt da casca $(9,1 \mathrm{~g})$ foi cromatografada sobre 80,0 g de gel de sílica, por eluição com hexano, AcOEt e MeOH, puros ou em misturas binárias de polaridade crescente. As frações obtidas, após análise em CCD, foram agrupadas em 15 subfrações. A subfração hexano/AcOEt (4:6) foi cromatografada, obtendo-se um precipitado amarelo, o qual foi filtrado e recristalizado em acetona, gerando o composto 5 (35 mg). A subfração hexano/AcOEt (3:7), após sucessivas cromatografias, forneceu a substância $\mathbf{6}(22 \mathrm{mg})$. A subfração AcOEt foi recromatografada, resultando por eluição com hexano/AcOEt (1:9) no isolamento da mistura de 7 e 8 (10 mg), enquanto da eluição com AcOEt obteve-se o composto 9 (35 mg). A fração $\mathrm{CHCl}_{3}$ do lenho $(6,2 \mathrm{~g})$ foi cromatografada sobre $60 \mathrm{~g}$ de gel de sílica utilizando hexano, AcOEt e $\mathrm{MeOH}$ como eluentes. As frações iniciais, provenientes da eluição com hexano e hexano/AcOEt $5 \%$, foram reunidas após análise em CCD, resultando em 1,3 g de um óleo amarelo alaranjado, o qual foi submetido à hidrólise alcalina $(\mathrm{MeOH} / \mathrm{NaOH} /$ refluxo) e, posteriormente, à reação de metilação ( $\mathrm{MeOH} / \mathrm{HCl} /$ refluxo). O produto reacional resultante $(340 \mathrm{mg})$ foi filtrado em gel de sílica, utilizando como eluente hexano/ $\mathrm{CHCl}_{3}(1: 1)$ e, subseqüentemente, analisado por CG/EM. A fração insaponificável (800 mg), após sucessivas cromatografias, forneceu por eluição com hexano/ $\mathrm{CHCl}_{3}$ (2:8) o composto $\mathbf{1 0}$ (15 mg). As demais frações, oriundas do tratamento cromatográfico da fração $\mathrm{CHCl}_{3}$, foram monito- radas por $\mathrm{CCD}$, identificando-se os metabólitos secundários 2, 3, 4 e 5. Seguindo procedimentos cromatográficos semelhantes aos descritos acima, foram identificados na fração AcOEt do lenho, os compostos 6, 7, 8 e 9 .

Excelsina (1): Sólido amorfo amarelado, p.f. $120-122{ }^{\circ} \mathrm{C}$; IV $(\mathrm{KBr}), v_{\max } \mathrm{cm}^{-1}: 2919,2851,1635,1511,1454,1192,1084$. EM (70 eV), m/z (int. rel.): 414 ([M] $\left.]^{+}, 75\right), 383$ (3), 207 (30), 191 (52), 179 (100), 165 (56), 152 (33). RMN ${ }^{1} \mathrm{H}\left(500 \mathrm{MHz}, \mathrm{CDCl}_{3}\right.$ ): 6,53 (d, $J=$ 1,0 Hz, H-2), 6,55 (d, $J=1,0 \mathrm{~Hz}, \mathrm{H}-6$ ), 4,71 (d, $J=4,2 \mathrm{~Hz}, \mathrm{H}-7$ ), 3,05 (m, H-8), 4,46 (dd, $J=9,1$ e 6,0 Hz, H-9a), 3,89 (dd, $J=9,1$ e 2,2 Hz, H-9b), 5,97 $\left(\mathrm{CH}_{2} \mathrm{O}_{2}\right), 3,92\left(\mathrm{CH}_{3} \mathrm{O}\right) . \mathrm{RMN}{ }^{13} \mathrm{C}(125 \mathrm{MHz}$, $\mathrm{CDCl}_{3}$ ): 136,1 (C-1), 100,4 (C-2), 144,0 (C-3), 135,0 (C-4), 149,5 (C-5), 105,9 (C-6), 86,2 (C-7), 54,7 (C-8), 72,2 (C-9), 101,9 $\left(\mathrm{CH}_{2} \mathrm{O}_{2}\right)$, $57,1\left(\mathrm{CH}_{3} \mathrm{O}\right)$.

Pinoresinol (2): Sólido amorfo amarelado, p.f. $125-127{ }^{\circ} \mathrm{C}$; IV (filme), $v_{\max } \mathrm{cm}^{-1}:$ 3403, 2963, 2868, 1605, 1516, 1460, 1272, 1036. EM (70 eV), m/z (int. rel.): 358 ([M] $\left.]^{+}, 42\right), 327$ (2), 180 (12), 163 (38), 151 (100), 137 (53), 124 (25). RMN ${ }^{1} \mathrm{H}$ (500 MHz, acetona$\left.\mathrm{d}_{6}\right): 6,99(\mathrm{~d}, J=1,8 \mathrm{~Hz}, \mathrm{H}-2), 6,79$ (d, $\left.J=8,0 \mathrm{~Hz}, \mathrm{H}-5\right), 6,83$ (dd, $J$ $=8,0$ e 1,8 Hz, H-6), 4,67 (d, $J=4,1 \mathrm{~Hz}), 3,08$ (m, H-8), 4,19 (dd, $J$ = 9,0 e 7,0 Hz, H-9a), 3,79 (dd, $J=9,0$ e 3,7 Hz, H-9b), 3,83 ( $\left.\mathrm{CH}_{3} \mathrm{O}\right)$, 7,69 (s, H0-4). RMN ${ }^{13} \mathrm{C}$ (125 MHz, acetona-d $\left.\mathrm{d}_{6}\right): 134,2$ (C-1), 110,7 (C-2), 148,4 (C-3), 146,9 (C-4), 115,7 (C-5), 119,7 (C-6), 86,7 (C7), 55,3 (C-8), 72,3 (C-9), 56,3 ( $\left.\mathrm{CH}_{3} \mathrm{O}\right)$.

Naringenina (5): Sólido amorfo amarelado, p.f. $253-254{ }^{\circ} \mathrm{C}$; IV $(\mathrm{KBr}), v_{\max } \mathrm{cm}^{-1}: 3298,1650,1604,1498,1156,1073 . \mathrm{RMN}^{1} \mathrm{H}(300$ $\mathrm{MHz}$, acetona-d $\left.)_{6}\right): 5,45$ (dd, $J=12,8$ e 3,0 Hz, H-2), 3,17 (dd, $J=$ 17,1 e $12,8 \mathrm{~Hz}, \mathrm{H}-3 \mathrm{a}$ ), 2,722 (dd, $J=17,1$ e 3,0 Hz, H-3b), 5,95 (s, H-6), 5,95 (s, H-8), 7,37 (d, $J=11,3$ Hz, H-2' e H-6'), 6,88 (d, $J=$ 11,3 Hz, H-3' e H-5'). RMN ${ }^{13} \mathrm{C}$ (75 MHz, acetona-d $)$ ): 80,0 (C-2), 43,5 (C-3), 197,3 (C-4), 165,3 (C-5), 96,9 (C-6), 167,4 (C-7), 95.9 (C-8), 164,4 (C-9), 103,3 (C-10), 130,8 (C-1'), 129,0 (C-2' e C-6'), 116,2 (C-3' e C-5'), 158,7 (C-4').

Aromadendrina (6): Sólido amorfo amarelado, p.f. $223-225{ }^{\circ} \mathrm{C}$; IV (KBr), $v_{\max } \mathrm{cm}^{-1}: 3465,1639,1600,1521,1172,1091 . \mathrm{RMN}{ }^{1} \mathrm{H}$ (300 MHz, CD ${ }_{3} \mathrm{OD}$ ): 5,01 (dd, $J=11,5 \mathrm{~Hz}, \mathrm{H}-2$ ), 4,57 (d, $J=11,5$ Hz, H-3), 5,93 (d, $J=2,1 \mathrm{~Hz}, \mathrm{H}-6$ ), 5,89 (d, $J=2,1 \mathrm{~Hz}, \mathrm{H}-8$ ), 7,35 (d, $J=8,5$ Hz, H-2' e H-6'), 6,83 (d, $J=8,5$ Hz, H-3' e H-5'). RMN ${ }^{13} \mathrm{C}$ (75 MHz, CD $\left.\mathrm{OD}\right): 85,3$ (C-2), 74,0 (C-3), 198,8 (C-4), 165,6 (C-5), 97,7 (C-6), 169,1 (C-7), 96,7 (C-8), 164,9 (C-9), 102,2 (C10), 129,6 (C-1'), 130,7 (C-2' e C-6'), 116,5 (C-3' e C-5'), 159,5 (C-4').

Ácido gálico (9): Cristais incolores, p.f. $252-253{ }^{\circ} \mathrm{C}$; IV (KBr), $\mathrm{V}_{\max } \mathrm{cm}^{-1}: 3466,1701,1649,1545,1262,1043$. RMN ${ }^{1} \mathrm{H}(300 \mathrm{MHz}$, CD $\mathrm{OD}$ ): 7.08 (s). RMN ${ }^{13} \mathrm{C}$ (75 MHz, CD $\mathrm{OD}$ ): 120,7 (C-1), 109,1 (C-2), 145,1 (C-3), 138,3 (C-4), 145,1 (C-5), 109, 1 (C-6), 169,1 (C7).

1-Dodecanoil-3,5-di(tetradecanoil)benzeno (10): Cristais amarelados, p.f. $36-39^{\circ} \mathrm{C}$; IV (KBr), $v_{\max } \mathrm{cm}^{-1}$ : 2924, 2854, 1694, 1591, 1462, 1170. EM (70 eV), m/z (int. rel.): 680 ([M] $\left.{ }^{+}, 10\right), 540$ (4), 469 (8), 344 (7), 204 (11), 189 (10), 161 (8). RMN ${ }^{1} \mathrm{H}$ (300 MHz, CD $\mathrm{OD}$ ): 8,69 (s, H-2, H-4 e H-6), 3,03 (t, $J=7,2 \mathrm{~Hz}, 3$ x CH 1,76 (m, $3 \times \mathrm{CH}_{2}$ b), 1,30 (s intenso, $2 \times 10 \mathrm{CH}_{2}+8 \mathrm{CH}_{2}$ ), 0,89 (t, 3 $\mathrm{x} \mathrm{CH}$ ) $) \cdot \mathrm{RMN}^{13} \mathrm{C}\left(75 \mathrm{MHz}, \mathrm{CD}_{3} \mathrm{OD}\right): 138,0(\mathrm{C}-1, \mathrm{C}-3$ e C-5), 131,0 (C-2, C-4 e C-6), 199,9 (C-7, C-7' e C-7'), 39,1 (3 x CH $2 \alpha), 32,1$ (3 $\left.x \mathrm{CH}_{2} \beta\right), 14.3\left(3 \times \mathrm{CH}_{3}\right)$.

\section{REFERÊNCIAS}

1. Poppendieck, H. H.; Flora Neotrópica, Cochlospermaceae, Organization for Flora Neotropica, The New York Botanical Garden: New York, 1981.

2. Presber, W.; Hegenscheid, B.; Hernandez-Alvarez, H.; Hermann D.; Brendel, C.; Acta Tropica 1992, 50, 331.

3. Aliyu, R.; Okoye, Z. S. C.; Shier W. T.; J. Ethnopharmacol. 1995, 48, 89. 
4. Diallo, B.; Vanhaelen, M.; Phytochemistry 1987, 26, 1491.

5. Esposito-Avella, M.; Brown, P.; Tejeira, I.; Buitrago, R.; Barrios, L.; Sanchez, C.; Ind. J. Crude Drug Res. 1985, 23, 17.

6. Beinot-Vical, F.; Valentin, A.; Da, B.; Dakuyo, Z.; Descamps, L.; Mallie, M.; J. Ethnopharmacol. 2003, 89, 111.

7. Lima, D. P.; Castro, M. S. A.; Mello, J. C. P.; Kassab, J. M. S.; Fitoterapia 1995, 66, 545.

8. Diallo, B.; Vanhaelen, M.; Vanhaelen-Fastré, R.; Konoshima T.; Kozuka M.; Tokuda, H.; J. Nat. Prod. 1989, 52, 879.

9. Diallo, B.; Vanhaelen-Fastré, R.; Vanhaelen, M.; Phytochemistry 1991, 30, 4153.

10. Achenbach, H.; Luschmann K.; Arch. Pharm. 1988, 321, 672.

11. Ballin, N. C.; Traore, M.; Tinto, H.; Sittie, A.; Molgaard, P.; Olsen, C. E.; Kharazmi, A.; Christensen, S. B.; J. Nat. Prod. 2002, 65, 1325.

12. Achenbach, H.; Blumm, E.; Waibel, R.; Tetrahedron Lett. 1989, 30, 3059.

13. Benoit-Vical, F.; Valentin, A.; Mallié, M.; Bastide, J. M.; Bessière, J. M.; Planta Med. 1999, 65, 378.

14. Russel, G. B.; Fenemore, P. G.; Phytochemistry 1973, 12, 1799.

15. Cowan, S.; Stewart, M.; Abbiw, D. K.; Latif, Z.; Sarker, S. D.; Nash, R. J.; Fitoterapia 2001, 72, 80.
16. Agrawal, P. K.; Thakur, R. S.; Bansal, M. C.; Carbon-13 NMR of Flavonoids, Elsevier Science Publicher B. V., 1989, p. 95-119.

17. Macari, P. A. T.; Emerenciano, V. P.; Ferreira, Z. M. G. S.; Quim. Nova 1990, 13, 260.

18. Poucher, C. T.; Behnke, J.; The Aldrich Library of ${ }^{13} \mathrm{C}$ and ${ }^{1} \mathrm{H}$ FT-NMR Spectra, 1a. ed., 1993, vol. 2.

19. Addae-Mensah, I.; Waibel, R.; Achenbach, H.; Liebgs Ann. Chem. 1985, 1284.

20. Craveiro, A. A.; Matos, F. J. A.; Alencar, J. W.; J. Nat. Prod. 1984, 47, 890.

21. Alencar, J. W.; Craveiro, A. A.; Matos, F. J. A.; Machado, M. I. L.; Quim. Nova 1990, 13, 282.

22. Stenhagen, E.; Abrahamson S.; McLafferty, F. W.; Registry of Mass Spectra Data, J. Wiley \& Sons: New York, 1974.

23. Adams, R. P.; Identification of Essential Oil Components by Gas Chromatography/Quadrupole Mass Spectroscopy, Allured Publishing Corporation: Illinois, 2001. 